\title{
Existence of Traveling Wave Solutions for Cholera Model
}

\author{
Tianran Zhang, ${ }^{1}$ Qingming Gou, ${ }^{2}$ and Xiaoli Wang ${ }^{1}$ \\ ${ }^{1}$ School of Mathematics and Statistics, Southwest University, Chongqing 400715, China \\ ${ }^{2}$ College of Mathematics \& Computer Science, Yangtze Normal University, Chongqing 408100, China
}

Correspondence should be addressed to Tianran Zhang; zhtr0123@126.com

Received 28 December 2013; Accepted 25 January 2014; Published 26 March 2014

Academic Editor: Kaifa Wang

Copyright (C) 2014 Tianran Zhang et al. This is an open access article distributed under the Creative Commons Attribution License, which permits unrestricted use, distribution, and reproduction in any medium, provided the original work is properly cited.

\begin{abstract}
To investigate the spreading speed of cholera, Codeço's cholera model (2001) is developed by a reaction-diffusion model that incorporates both indirect environment-to-human and direct human-to-human transmissions and the pathogen diffusion. The two transmission incidences are supposed to be saturated with infective density and pathogen density. The basic reproduction number $R_{0}$ is defined and the formula for minimal wave speed $c^{*}$ is given. It is proved by shooting method that there exists a traveling wave solution with speed $c$ for cholera model if and only if $c \geq c^{*}$.
\end{abstract}

\section{Introduction}

Cholera has been a serious threat to human health in the past and at present, which is an acute, diarrheal illness caused by infection of the intestine with the bacterium Vibrio cholera. An estimated 3-5 million cases and over 100,000 deaths occur each year around the world [1]. The cholera bacterium is usually found in water or food sources that have been contaminated by feces from a person infected with cholera. Cholera is most likely to be found and to spread in places with inadequate water treatment, poor sanitation, and inadequate hygiene. Therefore, cholera outbreaks have occurred in developing countries, for example, Iraq (20072008), Guinea Bissau (2008), Zimbabwe (2008-2009), Haiti (2010), Democratic Republic of Congo (2011-2012), and Sierra Leone (2012) [2].

To understand the propagation mechanism of cholera, many mathematical models were proposed, whose earlier one was established by Capasso and Paveri-Fontana [3] to study the 1973 cholera epidemic in the Mediterranean region as follows:

$$
\frac{d I}{d t}=g(B)-a_{22} I, \quad \frac{d B}{d t}=-a_{11} B+a_{12} I,
$$

where $B(t)$ and $I(t)$ denote the concentrations of the pathogen and the infective populations, respectively. In addition, Codeço [4] investigated the role of the aquatic pathogen in dynamics of cholera through the following susceptibleinfective-pathogen model:

$$
\begin{gathered}
\frac{d S}{d t}=n(H-S)-a \frac{S B}{K+B}, \quad \frac{d I}{d t}=a \frac{S B}{K+B}-r I, \\
\frac{d B}{d t}=e I-(m b-n b) B,
\end{gathered}
$$

where $S(t)$ is the susceptible individuals. In this model, human is divided into two groups: the susceptible group and the infective group. As pointed out in [4-8], bacterium Vibrio cholera can spread by direct human-to-human and indirect environment-to-human modes. To understand the complex dynamics of cholera, model (2) is extended by [8-15] and so forth.

In all previous models the influences of space distribution of human on the transmission of cholera are omitted. Cholera usually spreads in spatial wave [16]. Cholera bacteria live in rivers and interact with the plankton on the surface of the water [17]. When individuals drink contaminated water and are infected, they will release cholera bacteria through excretion [18]. Capasso et al. [19-23] developed model (1) by incorporating the bacterium diffusion in a bounded area and studied the existence and stability of solutions. To deeply investigate the interaction of transmission modes and bacterium diffusion, Bertuzzo et al. [24, 25] incorporated patchy structure into model (2) and supposed that pathogen 
in water could diffuse among these patches. Furthermore, Mari et al. [26] studied the influence of diffusion of both human and pathogen on cholera dynamics through a patchy model.

Infectious case is usually found firstly at some location and then spreads to other areas. Consequently, the most important question for cholera is what the spreading speed of cholera is. However, the above spatial models mainly focus on the stability of solutions not the spreading speed. Traveling wave solution is an important tool used to study the spreading speed of infectious diseases [27-29]. Based on Capasso's model (1), Zhao and Wang [30], Xu and Zhao [31], Jin and Zhao [32], and Hsu and Yang [33] studied the influences of pathogen diffusion on the spread speed of cholera.

The studies of traveling wave solutions of Capasso's model (1) incorporating pathogen diffusion provide insight into the spreading speed of cholera. However, some pieces of information are omitted, such as the interaction of direct human-tohuman and indirect environment-to-human transmissions. In this paper, a reaction-diffusion model with pathogen diffusion and both transmission paths is proposed by developing Codeço's model (2). Based on model (2) and ignoring the disease-related death, a general diffusive cholera model can be formulated as the following reaction-diffusion system:

$$
\begin{gathered}
\frac{\partial S}{\partial t}=b(N-S)-f(I) S-g(B) S \\
\frac{\partial I}{\partial t}=f(I) S+g(B) S-b I \\
\frac{\partial B}{\partial t}=d \frac{\partial^{2} B}{\partial x^{2}}+e I-m B
\end{gathered}
$$

where $S=S(x, t)$ and $I=I(x, t)$ denote the concentrations of susceptible and infected individuals, respectively, and $B=$ $B(x, t)$ is the concentration of the infectious agents. $N$ is the total human population, $b$ stands for the natural birth and death rate, $e$ denotes the contribution of each infected person to the concentration of cholera, and $m$ is the net death rate of vibrio cholera. $f(I)$ and $g(B)$ are the human-tohuman and environment-to-human transmission incidences, respectively. Similar to [10], we assume that $f(I)$ and $g(B)$ satisfy

(A1) $f(0)=0, f^{\prime}(I) \geq 0, f^{\prime \prime}(I) \leq 0$;

(A2) $g(0)=0, g^{\prime}(0)>0, g^{\prime}(B) \geq 0, g^{\prime \prime}(B) \leq 0$, and $g(B)$ is strictly monotonously increasing in $[0,+\infty)$.

It is easy to conclude that $f(I) \leq f^{\prime}(0) I, g(B) \leq g^{\prime}(0) B$, and $f(I) / I$ and $g(B) / B$ are nonincreasing. Obviously, hypotheses (A1) and (A2) imply that the two transmission paths are saturated. In Tian and Wang [10], $f(I)$ and $g(B)$ have the following expressions:

$$
f(I)=\beta_{1} I, \quad g(B)=\frac{\beta_{2} B}{K+B} .
$$

Obviously, as a special case, such selections satisfy (A1) and (A2).

Shooting method is very important in proving the existence of traveling wave solutions, which was proposed by
Dunbar $[34,35]$ and was applied to many models (e.g., [36$40])$. In this paper, the existence of traveling wave solutions of system (3) will be proved by shooting method and the formula for minimal wave speed will be given.

This paper is organized as follows. In next section, the main theorem and the formula for minimal wave speed will be given. In Section 3, the nonexistence of the traveling wave solutions for $c<c^{*}$ is proved by geometric method. Section 4 is devoted to shooting arguments and the construction of Wazewski set. In Section 5, we prove the existence of traveling wave solutions for $c>c^{*}$ and then give the existence of traveling wave solution for $c=c^{*}$ by limit arguments. The final section is devoted to the simulations.

\section{Main Results}

For convenience, we introduce dimensionless variables and parameters. By setting

$$
u_{1}=\frac{S}{b N}, \quad u_{2}=\frac{I}{b N}, \quad u_{3}=\frac{m}{e b N} B, \quad y=\frac{x}{\sqrt{d}},
$$

model (3) has the form

$$
\begin{gathered}
u_{1, t}=1-b u_{1}-f_{1}\left(u_{2}\right) u_{1}-g_{1}\left(u_{3}\right) u_{1}, \\
u_{2, t}=f_{1}\left(u_{2}\right) u_{1}+g_{1}\left(u_{3}\right) u_{1}-b u_{2}, \\
u_{3, t}=u_{3, y y}+m\left(u_{2}-u_{3}\right),
\end{gathered}
$$

where $f_{1}\left(u_{2}\right)=f\left(b N u_{2}\right)$ and $g_{1}\left(u_{3}\right)=g\left(e b N u_{3} / m\right)$.

Denote $R_{0}=\left[f_{1}^{\prime}(0)+g_{1}^{\prime}(0)\right] / b^{2}$, which is the basic reproduction number of (6). Then hypotheses (A1) and (A2) imply that system (6) has two nonnegative constant solutions $P_{1}(1 / b, 0,0)$ and $P_{2}\left(1 / b-u^{*}, u^{*}, u^{*}\right)$ if and only if $R_{0}>1$, where $u^{*}$ is the only one positive root of equation

$$
\left[f_{1}\left(u^{*}\right)+g_{1}\left(u^{*}\right)\right]\left(\frac{1}{b}-u^{*}\right)=b u^{*}
$$

and $0<u^{*}<1 / b$. Biologically, $P_{1}$ corresponds to disease-free equilibrium and $P_{2}$ corresponds to endemic equilibrium. To study the spreading wave of cholera, it is assumed that $R_{0}>1$ holds in this paper; that is

$$
f_{1}^{\prime}(0)+g_{1}^{\prime}(0)>b^{2} .
$$

A traveling wave solution of system (6) with speed $c$ is a nonnegative solution of the form

$$
\begin{gathered}
u_{1}(y, t)=u_{1}(s), \quad u_{2}(y, t)=u_{2}(s), \\
u_{3}(y, t)=u_{3}(s), \quad s=y+c t .
\end{gathered}
$$

Substituting traveling profile $\left(u_{1}(s), u_{2}(s), u_{3}(s)\right)$ into system (6) yields the following equations:

$$
\begin{gathered}
c u_{1}^{\prime}=1-b u_{1}-f_{1}\left(u_{2}\right) u_{1}-g_{1}\left(u_{3}\right) u_{1}, \\
c u_{2}^{\prime}=f_{1}\left(u_{2}\right) u_{1}+g_{1}\left(u_{3}\right) u_{1}-b u_{2}, \\
c u_{3}^{\prime}=u_{3}^{\prime \prime}+m\left(u_{2}-u_{3}\right),
\end{gathered}
$$


where $/$ denotes $d / d s$. To investigate invasion question by cholera, we will study the positive solutions of (10) such that

$$
\begin{gathered}
\left(u_{1}(+\infty), u_{2}(+\infty), u_{3}(+\infty)\right)=\left(\frac{1}{b}-u^{*}, u^{*}, u^{*}\right), \\
\left(u_{1}(-\infty), u_{2}(-\infty), u_{3}(-\infty)\right)=\left(\frac{1}{b}, 0,0\right) .
\end{gathered}
$$

Before giving the main theorem, we introduce the equation for minimal wave speed

$$
\Delta(c):=b_{3} c^{6}+b_{2} c^{4}+b_{1} c^{2}+b_{0}=0,
$$

where

$$
\begin{gathered}
\epsilon=f_{1}^{\prime}(0)-b^{2}, \\
b_{3}=b^{2} \epsilon^{2}+2 b^{3} m\left(f_{1}^{\prime}(0)+g_{1}^{\prime}(0)-b^{2}\right) \\
+2 b^{3} m g_{1}^{\prime}+b^{4} m^{2}, \\
b_{2}=-2 b \epsilon^{3}+2 b^{2} m \epsilon^{2}+\left(8 b^{3} m^{2}-6 b^{2} m g_{1}^{\prime}\right) \epsilon \\
+4 m^{3} b^{4}+18 b^{3} m^{2} g_{1}^{\prime}(0), \\
b_{1}=\epsilon^{4}-8 m b \epsilon^{3}-\left(8 b^{2} m^{2}+6 b m g_{1}^{\prime}\right) \epsilon^{2} \\
-36 b^{2} m^{2} g_{1}^{\prime}(0) \epsilon-27 m^{2} b^{2} g_{1}^{\prime}(0)^{2}, \\
b_{0}=4 m\left(b^{2}-f_{1}^{\prime}(0)\right)^{3}\left(b^{2}-f_{1}^{\prime}(0)-g_{1}^{\prime}(0)\right) .
\end{gathered}
$$

Theorem 1. There exists a constant $c^{*}>0$ which is the greatest positive root of (12). When $c \geq c^{*}$, system (6) has a traveling wave solution satisfying boundary condition (11). When $0<$ $c<c^{*}$, system (6) has no traveling wave solutions satisfying boundary condition (11).

\section{Nonexistence of Traveling Wave Solutions for $c<c^{*}$}

From (10), we have

$$
\left[u_{1}(s)+u_{2}(s)\right]^{\prime}=\frac{\left[1-b\left(u_{1}(s)+u_{2}(s)\right)\right]}{c} .
$$

Consequently, if $u_{1}(0)+u_{2}(0) \neq 1 / b$, then

$$
\left|u_{1}(s)+u_{2}(s)\right| \longrightarrow \infty \quad \text { when } s \longrightarrow-\infty \text {. }
$$

Hence, the traveling profile $\left(u_{1}(s), u_{2}(s), u_{3}(s)\right)$ with boundary condition (11) must satisfy

$$
u_{1}(s)+u_{2}(s)=\frac{1}{b} \quad \text { for any } s \in R .
$$

Therefore, to study traveling wave solutions we assume (16) satisfies. Setting $u_{3}^{\prime}=z$ in system (10) and noticing (16), it follows

$$
\begin{gathered}
u_{2}^{\prime}=\frac{\left[\left(f_{1}\left(u_{2}\right)+g_{1}\left(u_{3}\right)\right)\left((1 / b)-u_{2}\right)-b u_{2}\right]}{c}, \\
u_{3}^{\prime}=z \\
z^{\prime}=c z+m\left(u_{3}-u_{2}\right) .
\end{gathered}
$$

If $u_{1}(s)=0$, then $u_{1}^{\prime}(s)=1 / c>0$ by system $(10)$. Therefore, we suppose $u_{1}(s)=1 / b-u_{2}(s)>0$ for any $s$; that is, $u_{2}(s)<1 / b$.

Obviously, system (17) has two equilibria $E_{1}(0,0,0)$ and $E_{2}\left(u^{*}, u^{*}, 0\right)$. A profile solution of (10) which satisfies boundary condition (11) corresponds to the positive solution $\left(u_{2}(s), u_{3}(s), z(s)\right)$ of system (17) which satisfies

$$
u(+\infty)=\left(u_{2}^{*}, u_{2}^{*}, 0\right), \quad u(-\infty)=(0,0,0),
$$

where $u(s)=\left(u_{2}(s), u_{3}(s), z(s)\right)$. Therefore, to study the solutions of (10), it is sufficient to study those of system (17) satisfying boundary condition (18).

Firstly, we investigate the dynamics near $E_{1}$. Simple calculations show that the characteristic equation of the linearization of system (17) at $E_{1}$ is

$$
H(\lambda)=\lambda^{3}+a_{2} \lambda^{2}+a_{1} \lambda+a_{0}=0,
$$

where

$$
\begin{gathered}
a_{0}=\frac{m\left(f_{1}^{\prime}(0)+g_{1}^{\prime}(0)-b^{2}\right)}{b c}, \quad a_{1}=\frac{f_{1}^{\prime}(0)-b^{2}-m b}{b}, \\
a_{2}=\frac{b^{2}-f_{1}^{\prime}(0)-b c^{2}}{b c} .
\end{gathered}
$$

Because $a_{0}>0$ (19) has a negative real root, which is denoted by $\lambda_{3}$. Let $\lambda_{1}$ and $\lambda_{2}$ be the other two eigenvalues of (19) and suppose that $\operatorname{Re} \lambda_{1} \geq \operatorname{Re} \lambda_{2}$. To investigate the distribution of roots of (19), denote

$$
p=a_{1}-\frac{a_{2}^{2}}{3}, \quad q=\frac{2 a_{2}^{3}}{27}-\frac{a_{1} a_{2}}{3}+a_{0}, \quad \Delta_{0}=\frac{q^{2}}{4}+\frac{p^{3}}{27}
$$

and introduce the following lemma [41].

Lemma 2. (a) If $\Delta_{0}>0$, (19) has one real root and two nonreal complex conjugate roots.

(b) If $\Delta_{0}=0$, (19) has a multiple root and all its roots are real.

(c) If $\Delta_{0}<0$, (19) has three distinct real roots.

Direct calculations show that $\Delta_{0}=-\Delta /\left(108 b^{4} c^{4}\right)$, where $\Delta$ is defined by (12).

Lemma 3. (a) The real parts of $\lambda_{1}$ and $\lambda_{2}$ are positive.

(b) Assume $f_{1}^{\prime}(0) \leq b^{2}$. Then, there exists $c^{*}>0$ which is the only positive root of $\Delta(c)=0$. When $c \geq c^{*}, \lambda_{1}$, and $\lambda_{2}$ are real. When $0<c<c^{*}, \lambda_{1}$, and $\lambda_{2}$ are complex and nonreal.

(c) Assume that $f_{1}^{\prime}(0)>b^{2}$. Then, there exist two positive constants $c_{1}^{*}<c^{*}$ which are all positive roots of $\Delta(c)=0 . \lambda_{1}$ and $\lambda_{2}$ are complex and nonreal if and only if $c_{1}^{*}<c<c^{*}$. If $c>c^{*}$, then $\lambda^{*}<\lambda_{2}<\lambda_{1}$; if $0<c \leq c_{1}^{*}$, then $\lambda_{2} \leq \lambda_{1}<\lambda^{*}$, where $\lambda^{*}=\left(f_{1}^{\prime}(0)-b^{2}\right) /(b c)$.

(d) $\lambda_{1}=\lambda_{2}$ if and only if $c=c^{*}$ or $c_{1}^{*}$.

Proof. Suppose $\lambda=\beta i \neq 0$ is the root of (19). Substituting $\lambda=\beta i$ into (19) and comparing real and imaginary parts 
show that $a_{1}=\beta^{2}>0$ and $a_{0}=a_{1} a_{2}$. Since $a_{0}>0$, then $a_{2}>0$. However, it is impossible that $a_{1}>0$ and $a_{2}>0$ by the expressions of $a_{1}$ and $a_{2}$. Therefore, the real parts of $\lambda_{1}$ and $\lambda_{2}$ are not zero. Furthermore, since it is impossible that $a_{1}>0$ and $a_{2}>0$, Routh-Hurwitz theorem implies that it is impossible that the real parts of both $\lambda_{1}$ and $\lambda_{2}$ are negative. Consequently, there are two cases: (i) $\lambda_{1}$ and $\lambda_{2}$ are complex conjugate roots with positive real parts; (ii) $\lambda_{1}$ and $\lambda_{2}$ are real and at least one is positive. However, Descartes' rule of signs shows that the number of positive roots of (19) is zero or two. Thus, if case (ii) is true, both of $\lambda_{1}$ and $\lambda_{2}$ are real and positive. Therefore, (a) is proved.

In this paragraph, we consider the case $f_{1}^{\prime}(0) \leq b^{2}$. Firstly, suppose that $f_{1}^{\prime}(0)<b^{2}$. Obviously, $b_{0}<0$ and $b_{3}>0$. By the expression of $b_{2}$, we have

$$
\begin{aligned}
b_{2}= & -2 b \epsilon^{3}+2 b^{2} m \epsilon^{2}-6 b^{2} m g_{1}^{\prime} \epsilon \\
& +8 b^{3} m^{2}\left(f_{1}^{\prime}(0)+g_{1}^{\prime}(0)-b^{2}\right)+4 m^{3} b^{4}+10 b^{3} m^{2} g_{1}^{\prime}(0) \\
> & 0
\end{aligned}
$$

since $\epsilon=f_{1}^{\prime}(0)-b^{2}<0$. Now, assume $f_{1}^{\prime}(0)=b^{2}$; that is, $\epsilon=0$. Then. $b_{3}>0, b_{2}>0, b_{1}<0$, and $b_{0}=0$. Then, if $f_{1}^{\prime}(0) \leq b^{2}$, Descartes' rule of signs shows that there exists $c^{*}>0$ which is the only positive root of $\Delta(c)=0$, where $\Delta(c)<0$ for $0<c<c^{*}$ and $\Delta(c)>0$ for $c>c^{*}$. Using Lemma 2 completes the proof of (b).

Suppose that $f_{1}^{\prime}(0)>b^{2}$ in this paragraph and, thus, $\epsilon>0$. Calculations show that

$$
\begin{gathered}
H\left(\lambda^{*}\right)=\frac{m g_{1}^{\prime}}{b c}>0, \\
H^{\prime}\left(\lambda^{*}\right)=\frac{\epsilon^{2}-b c^{2} \epsilon-m b^{2} c^{2}}{b^{2} c^{2}}
\end{gathered}
$$

and that $H^{\prime}(\lambda)=0$ has two roots $\lambda_{1}^{*}$ and $\lambda_{2}^{*}$, where

$$
\begin{aligned}
& \lambda_{1}^{*}=\frac{b c^{2}+\epsilon+\sqrt{b^{2} c^{4}+\left(3 m b^{2}-b \epsilon\right) c^{2}+\epsilon^{2}}}{3 b c}, \\
& \lambda_{2}^{*}=\frac{b c^{2}+\epsilon-\sqrt{b^{2} c^{4}+\left(3 m b^{2}-b \epsilon\right) c^{2}+\varepsilon^{2}}}{3 b c},
\end{aligned}
$$

and $\lambda_{1}^{*}>\lambda_{2}^{*}$. By letting $c_{0} \triangleq \epsilon / \sqrt{b \epsilon+m b^{2}}$ and using trivial calculations, we get (see Figure 1)

$$
\begin{aligned}
& \lambda^{*}=\lambda_{1}^{*} \Longleftrightarrow c=c_{0} \Longleftrightarrow H^{\prime}\left(\lambda^{*}\right)=0, \\
& \lambda^{*}>\lambda_{1}^{*} \Longleftrightarrow c<c_{0} \Longleftrightarrow H^{\prime}\left(\lambda^{*}\right)>0, \\
& \lambda^{*}<\lambda_{1}^{*} \Longleftrightarrow c>c_{0} \Longleftrightarrow H^{\prime}\left(\lambda^{*}\right)<0 .
\end{aligned}
$$

Therefore, if $c=c_{0}$, then $H\left(\lambda_{1}^{*}\right)=H\left(\lambda^{*}\right)>0$. Since $\lambda_{1}^{*}$ is the only minimum-value point of $H(\lambda)$, and then $H(\lambda)>0$ for any $\lambda>0$ and both of $\lambda_{1}$ and $\lambda_{2}$ are not real. Lemma 2 shows that $\Delta\left(c_{0}\right)<0$. Thus, since $b_{0}>0$ and $b_{3}>0$, there exist two positive roots $c_{1}^{*}<c^{*}$ for equation $\Delta(c)=0$ such that $c_{1}^{*}<c_{0}<c^{*}$. Then, using (25) and Lemma 2 completes the proof of (c) and (d).

Direct calculations show that corresponding eigenvectors of eigenvalue $\lambda_{i}$ are

$$
e_{i}=\left(1-\frac{\lambda_{i}\left(\lambda_{i}-c\right)}{m}, 1, \lambda_{i}\right),
$$

where $i=1,2,3$. Since

$$
\begin{aligned}
H\left(\lambda_{i}\right) & =-m\left[1-\frac{\lambda_{i}\left(\lambda_{i}-c\right)}{m}\right]\left[\lambda_{i}-\frac{f_{1}^{\prime}(0)-b^{2}}{b c}\right]+\frac{m g_{1}^{\prime}}{b c} \\
& =0
\end{aligned}
$$

and thus

$$
1-\frac{\lambda_{i}\left(\lambda_{i}-c\right)}{m}=\frac{g_{1}^{\prime}(0)}{b c \lambda_{i}+b^{2}-f_{1}^{\prime}(0)}=\frac{g_{1}^{\prime}(0)}{b c\left(\lambda_{i}-\lambda^{*}\right)} .
$$

Then, we have the following lemma.

Lemma 4. If $0<c<c^{*}$, there exist no traveling wave solutions which satisfy boundary condition (11).

Proof. Assume that $f_{1}^{\prime}(0) \leq b^{2}$ and $0<c<c^{*}$. Then, (b) of Lemma 3 implies that $\lambda_{1}$ and $\lambda_{2}$ are complex conjugate eigenvalues and there exits locally unstable manifold $\mathscr{W}^{u}$ and locally stable manifold $\mathscr{W}^{s}$. If a solution of (17) tends to $E_{1}$ when $s \rightarrow-\infty$, then it will be spiral on $\mathscr{W}^{u}$. By the structures of $e_{1}$ and $e_{2}, u_{2}(s)<0$ at some time $s<0$, which shows that there exist no traveling wave solutions departing from $E_{1}$.

Suppose that $f_{1}^{\prime}(0)>b^{2}$. If $c_{1}^{*}<c<c^{*}$, (c) of Lemma 3 shows that $\lambda_{1}$ and $\lambda_{2}$ are complex conjugate eigenvalues and similar arguments to that of previous paragraph finish the proof. If $0<c \leq c_{1}^{*}$, (c) of Lemma 3 shows that $\lambda_{1}$ and $\lambda_{2}$ are real; however, $\lambda_{2} \leq \lambda_{1}<\lambda^{*}$. If a solution of (17) tends to $E_{1}$ when $s \rightarrow-\infty$, structures of $e_{1}$ and $e_{2}$ indicate that there is an $s<0$ such that $u_{2}(s)<0$. The proof is completed.

From Section 4 to Section 5.2, we suppose that $c>c^{*}$, which implies $\lambda^{*}<\lambda_{2}<\lambda_{1}$.

\section{Shooting Method and Wazewski Set}

To prove the existence of traveling wave, shooting method developed by Dunbar [34] is used. Firstly, we give the shooting arguments.

Consider the differential equation

$$
\frac{d y}{d s}=f(y)
$$

where $f(y)$ from $R^{n}$ to $R^{n}$ satisfies Lipschitz condition about $y$. Let $y\left(s ; y_{0}\right)$ denote the unique solution of (29) with initial 


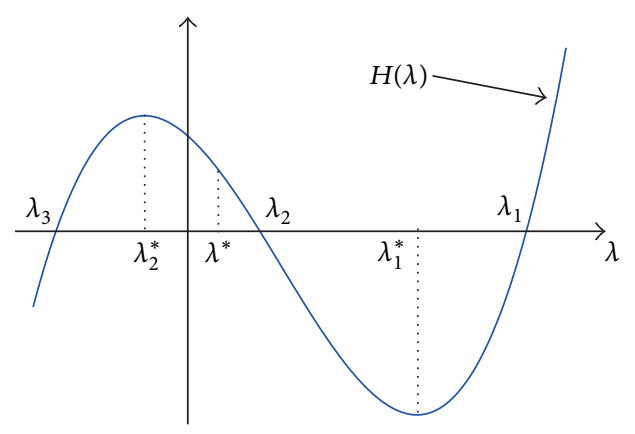

(a)

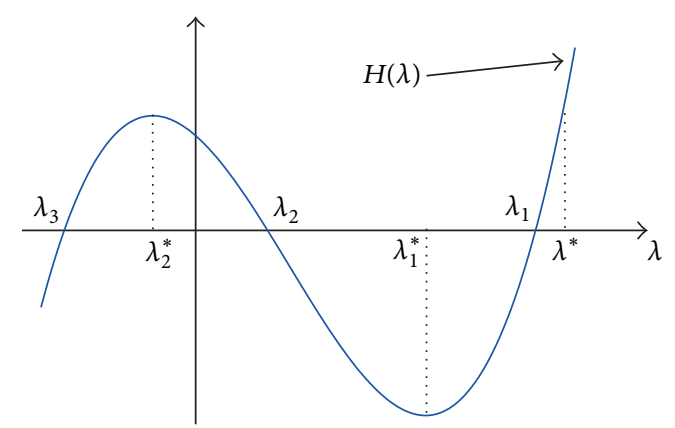

(b)

FIGURE 1: Distribution of eigenvalues of (19) when $f_{1}^{\prime}(0)>b^{2}$, (a) for $c>c^{*}$ and (b) for $c<c_{1}^{*}$.

value $y(0)=y_{0}$. It is convenient to give the notations $y_{0} \cdot s \triangleq$ $y\left(s ; y_{0}\right)$ and $y_{0} \cdot S \triangleq\left\{y_{0} \cdot s \mid s \in S \subset R\right\}$. To describe the shooting method (or Wazewski theorem), some definitions are necessary.

Definition 5. (a) For $W \subseteq R^{n}$, define immediate exit set $W^{-}$ of $W$ as

$$
W^{-} \triangleq\left\{y_{0} \in W \mid \forall s>0, y_{0} \cdot[0, s) \nsubseteq W\right\} .
$$

(b) For $\Sigma \subseteq W$, let $\Sigma^{0} \triangleq\left\{y_{0} \in \Sigma \mid \exists s_{0}>0\right.$ such that $y_{0}$. $\left.s_{0} \notin W\right\}$.

(c) Given $y_{0} \in \Sigma^{0}$, define exit time $T\left(y_{0}\right)$ of $y_{0}$ by

$$
T\left(y_{0}\right) \triangleq \sup \left\{s \mid y_{0} \cdot[0, s) \subseteq W\right\} .
$$

Then, Wazewski theorem is formulated as follows.

Lemma 6 (see [34]). Suppose that

(1) if $y_{0} \in \Sigma$ and $y_{0} \cdot[0, s] \subseteq \mathrm{cl}(W)$, then $y_{0} \cdot[0, s] \subseteq W$.

(2) If $y_{0} \in \Sigma, y_{0} \cdot s \in W$ and $y_{0} \cdot s \notin W^{-}$, then there exists an open set $V_{s}$ about $y_{0} \cdot s$ disjoint from $W^{-}$.

(3) If $\Sigma=\Sigma^{0}, \Sigma$ is compact and $\Sigma$ intersects a trajectory of (29) only once.

Then, the mapping $H\left(y_{0}\right)=y_{0} \cdot T\left(y_{0}\right)$ is a homeomorphism from $\Sigma$ to its image on $W^{-}$.

A set $W \subseteq R^{n}$ satisfying conditions (1) and (2) of Lemma 6 is called a Wazewski set. In the following, we first construct the Wazewski set $W$. Fundamental idea to construct a Wazewski set is that the characteristic vectors corresponding eigenvalues with positive real parts should be removed from $W$ and that those characteristic vectors corresponding eigenvalues with negative real parts should be included. Therefore, we set

$$
W=\mathbb{R}^{3} \backslash(P \cup Q),
$$

where

$$
\begin{gathered}
P=\left\{\left(u_{1}, u_{2}, u_{3}, z\right): u_{3}>u_{2}>u^{*}, z>0\right\}, \\
Q=\left\{\left(u_{1}, u_{2}, u_{3}, z\right): 0<u_{3}<u_{2}<u^{*}, z<0\right\} .
\end{gathered}
$$

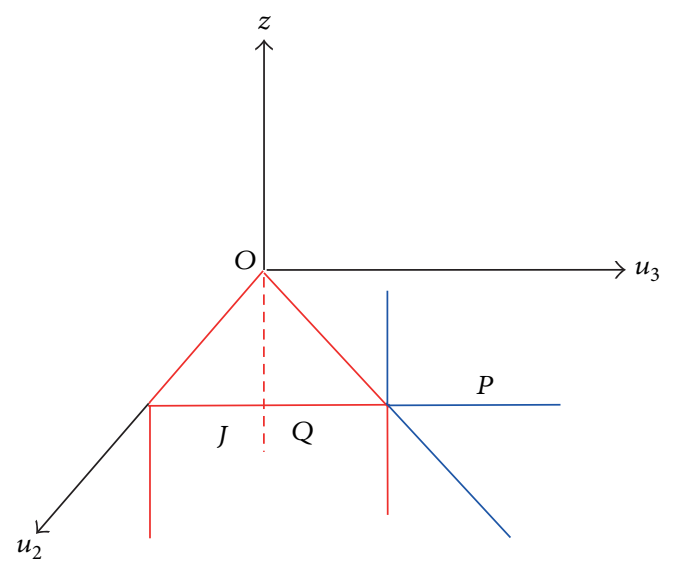

Figure 2: The construction of $W$ and $W^{-}$.

It is obvious that $\partial W=\partial P \cup \partial Q$. Firstly, we give the construction of $W^{-}$, which is described in Figure 2.

Lemma 7. The construction of $W^{-}$is as follows:

$$
W^{-}=\partial W \backslash\left(J \cup E_{2}\right),
$$

where $J=\left\{\left(u_{2}, u_{3}, z\right): 0 \leq u_{2} \leq u^{*}, u_{3}=0, z \leq 0\right\}$.

Proof. It is enough to analyze the behavior of solution on $\partial P \cup \partial Q$. We only study $\partial Q$ and omit the proof of $\partial P$ since the analysis of $\partial P$ is similar to that of $\partial Q$ and is simpler. In the process of this proof, we use some notations to simplify the proof. Set

$$
\begin{gathered}
u_{i}^{\prime}=\left.\frac{d u_{i}}{d s}\right|_{\left(u_{2}, u_{3}, z\right) \in \partial Q}, \quad z^{\prime}=\left.\frac{d z}{d s}\right|_{\left(u_{2}, u_{3}, z\right) \in \partial Q}, \quad i=2,3, \\
h\left(u_{2}\right)=\left[\frac{f_{1}\left(u_{2}\right)}{u_{2}}+\frac{g_{1}\left(u_{2}\right)}{u_{2}}\right]\left(\frac{1}{b}-u_{2}\right)-b .
\end{gathered}
$$

From hypotheses (A1) and (A2), we find that $f_{1}\left(u_{2}\right) / u_{2}$ and $g_{1}\left(u_{2}\right) / u_{2}$ are monotonously decreasing, $h\left(u_{2}\right)$ is strictly monotonously decreasing for $u_{2} \in(0,1 / b)$, and $u^{*}$ is the only positive root of $h\left(u_{2}\right)=0$. The set $\partial Q$ is classified into two cases according to variable $z$. 
(a) Case $z<0$. This case is classified as follows.

(1) Case $0=u_{3}<u_{2}<u^{*}$. Then $u_{3}^{\prime}=z<0$ and the solution of (17) will enter $\operatorname{int}(W)$.

(2) Case $0<u_{3}=u_{2}<u^{*}$. Then

$$
\left(u_{3}-u_{2}\right)^{\prime}=\frac{z-h\left(u_{2}\right) u_{2}}{c}<0 .
$$

The solution of (17) will enter $Q$.

(3) Case $0<u_{3}<u_{2}=u^{*}$. Then

$$
\begin{aligned}
u_{2}^{\prime} & =\frac{\left[\left(f_{1}\left(u^{*}\right) / u^{*}+g_{1}\left(u_{3}\right) / u^{*}\right)\left(1 / b-u^{*}\right)-b\right] u^{*}}{c} \\
& <\frac{h\left(u^{*}\right) u^{*}}{c}=0 .
\end{aligned}
$$

The solution of (17) will enter $Q$.

(4) Case $0=u_{3}=u_{2}<u^{*}$. Then $u_{3}^{\prime}=z<0$ and the solution of (17) will enter int $(W)$.

(5) Case $0=u_{3}$ and $u_{2}=u^{*}$. The solution of (17) will enter $\operatorname{int}(W)$.

(6) Case $u_{3}=u_{2}=u^{*}$. Then $u_{2}^{\prime}=0$,

$$
\begin{gathered}
u_{2}^{\prime \prime}=\left[\left(f_{1}^{\prime}(0)\left(u^{*}\right) u_{2}^{\prime}+g_{1}^{\prime}(0)\left(u^{*}\right) z\right)\left(\frac{1}{b}-u^{*}\right)\right. \\
\left.-\left(f_{1}\left(u^{*}\right)+g_{1}\left(u^{*}\right)\right) u_{2}^{\prime}-b\right] \times(c)^{-1} \\
=\frac{\left[g_{1}^{\prime}(0)\left(u^{*}\right) z\left((1 / b)-u^{*}\right)-b\right]}{c}<0,
\end{gathered}
$$

and $\left(u_{3}-u_{2}\right)^{\prime}=z<0$. Therefore, the solution of (17) will enter $Q$.

(b) Case $z=0$. This case is classified as follows.

(1) Case $0<u_{3}<u_{2}<u^{*}$. Then $z^{\prime}=m\left(u_{3}-u_{2}\right)<0$ and the solution of (17) will enter $Q$.

(2) Case $0=u_{3}<u_{2}<u^{*}$. Then $u_{3}^{\prime}=z=0$, $u_{3}^{\prime \prime}=z^{\prime}=-m u_{2}<0$. The solution of (17) will enter int $(W)$.

(3) Case $0<u_{3}=u_{2}<u^{*}$. Then $\left(u_{3}-u_{2}\right)^{\prime}=$ $-h\left(u_{2}\right) u_{2} / c<0, z^{\prime}=0$, and $z^{\prime \prime}=c z^{\prime}+$ $m\left(u_{3}-u_{2}\right)^{\prime}<0$. The solution of (17) will enter Q.

(4) Case $0<u_{3}<u_{2}=u^{*}$. Then $u_{2}^{\prime}<0$ and $z^{\prime}=$ $m\left(u_{3}-u_{2}\right)<0$. The solution of (17) will enter Q.

(5) Case $0=u_{3}=u_{2}<u^{*}$. In this case, $(0,0,0)$ is equilibrium and is constant.

(6) Case $0=u_{3}$ and $u_{2}=u^{*}$. Then $u_{3}^{\prime}=z=0$ and $u_{3}^{\prime \prime}=z^{\prime}=-m u_{2}<0$. The solution of (17) will enter $\operatorname{int}(W)$.

(7) Case $u_{3}=u_{2}=u^{*}$. Then $\left(u^{*}, u^{*}, 0\right)$ is equilibrium and is constant.

The proof is completed.

\section{Existence of Traveling Wave \\ Solution for $c \geq c^{*}$}

In this section, we prove the existence of traveling wave solution for $c \geq c^{*}$. Firstly, we study the behaviors of solutions near $E_{1}$.

\subsection{Behaviors of Solutions Near $E_{1}$}

Lemma 8. Suppose $\left(u_{2}(s), u_{3}(s), z(s)\right)$ is a solution of (17) satisfying initial conditions

$$
z(0)>k u_{3}(0), \quad u_{3}(0)>\frac{b c k+b^{2}-f_{1}^{\prime}(0)}{g_{1}^{\prime}(0)} u_{2}(0)>0
$$

where $k=\left(\lambda_{1}+\lambda_{2}\right) / 2$. Then, for everys $>0$, we have

$$
z(s)>k u_{3}(s), \quad u_{3}(s)>\frac{b c k+b^{2}-f_{1}^{\prime}(0)}{g_{1}^{\prime}(0)} u_{2}(s)>0 .
$$

Proof. From Lemma 3, we have $\left(b c k+b^{2}-f_{1}^{\prime}(0)\right) / g_{1}^{\prime}(0)>0$. To finish the proof, it is sufficient to prove that the set

$$
\Psi=\left\{\left(u_{2}, u_{3}, z\right): z>k u_{3}, u_{3}>\frac{b c k+b^{2}-f_{1}^{\prime}(0)}{g_{1}^{\prime}(0)} u_{2}>0\right\}
$$

is positively invariant. It is obvious that

$$
\partial \Psi=\partial \Psi_{1} \cup \partial \Psi_{2} \cup \partial \Psi_{3} \cup E_{1},
$$

where

$$
\begin{gathered}
\partial \Psi_{1}=\left\{\left(u_{2}, u_{3}, z\right): z=k u_{3}, u_{3} \geq \frac{b c k+b^{2}-f_{1}^{\prime}(0)}{g_{1}^{\prime}(0)} u_{2}>0\right\}, \\
\partial \Psi_{2}=\left\{\left(u_{2}, u_{3}, z\right): z>k u_{3}, u_{3}=\frac{b c k+b^{2}-f_{1}^{\prime}(0)}{g_{1}^{\prime}(0)} u_{2} \geq 0\right\}, \\
\partial \Psi_{3}=\left\{\left(u_{2}, u_{3}, z\right): z \geq k u_{3}, u_{3}>u_{2}=0\right\} .
\end{gathered}
$$

Suppose that $\left(u_{2}\left(s_{0}\right), u_{3}\left(s_{0}\right), z\left(s_{0}\right)\right) \in \partial \Psi_{1}$. Then, $z\left(s_{0}\right)=$ $k u_{3}\left(s_{0}\right)$ and

$$
\begin{aligned}
\frac{d}{d s} & {\left[z(s)-k u_{3}(s)\right]_{s=s_{0}} } \\
& =c z\left(s_{0}\right)+m\left[u_{3}\left(s_{0}\right)-u_{2}\left(s_{0}\right)\right]-k z\left(s_{0}\right) \\
& =(c-k) z\left(s_{0}\right)+m\left[u_{3}\left(s_{0}\right)-u_{2}\left(s_{0}\right)\right] \\
& =(c-k) k u_{3}\left(s_{0}\right)+m\left[u_{3}\left(s_{0}\right)-u_{2}\left(s_{0}\right)\right] \\
& =[(c-k) k+m] u_{3}\left(s_{0}\right)-m u_{2}\left(s_{0}\right) \\
& \geq\left\{[(c-k) k+m] \frac{b c k+b^{2}-f_{1}^{\prime}(0)}{g_{1}^{\prime}(0)}-m\right\} u_{2}\left(s_{0}\right) \\
& =-\frac{b c}{g_{1}^{\prime}(0)} H(k) u_{2}\left(s_{0}\right)>0 .
\end{aligned}
$$


The last inequality is given since $\lambda_{2}<k<\lambda_{1}$. Suppose that $\left(u_{2}\left(s_{0}\right), u_{3}\left(s_{0}\right), z\left(s_{0}\right)\right) \in \partial \Psi_{2}$. If $u_{2}\left(s_{0}\right)>0$, then

$$
\begin{aligned}
& \frac{d}{d s}\left[u_{3}(s)-\frac{b c k+b^{2}-f_{1}^{\prime}(0)}{g_{1}^{\prime}(0)} u_{2}(s)\right]_{s=s_{0}} \\
& =\left\{z-\frac{b c k+b^{2}-f_{1}^{\prime}(0)}{g_{1}^{\prime}(0)} \cdot \frac{1}{c}\right. \\
& \left.\times\left[\left(f_{1}\left(u_{2}\right)+g_{1}\left(u_{3}\right)\right)\left(\frac{1}{b}-u_{2}\right)-b u_{2}\right]\right\}_{s=s_{0}} \\
& >\left\{k u_{3}-\frac{b c k+b^{2}-f_{1}^{\prime}(0)}{c g_{1}^{\prime}}\right. \\
& \left.\times\left[\frac{f_{1}^{\prime}(0) u_{2}+g_{1}^{\prime}(0) u_{3}}{b}-b u_{2}\right]\right\}_{s=s_{0}} \\
& =\left\{\frac{b^{2}-f_{1}^{\prime}(0)}{b c g_{1}^{\prime}}\left[\left(b c k+b^{2}-f_{1}^{\prime}(0)\right) u_{2}-g_{1}^{\prime}(0) u_{3}\right]\right\}_{s=s_{0}} \\
& =0 .
\end{aligned}
$$

If $u_{2}\left(s_{0}\right)=0$, we have

$$
\begin{aligned}
& \frac{d}{d s}\left[u_{3}(s)-\frac{b c k+b^{2}-f_{1}^{\prime}(0)}{g_{1}^{\prime}(0)} u_{2}(s)\right]_{s=s_{0}} \\
& \quad=z\left(s_{0}\right)>0 .
\end{aligned}
$$

Consequently, the solution of system (17) departing from $\Psi$ cannot intersect $\partial \Psi_{1} \cup \partial \Psi_{2}$. If $\left(u_{2}\left(s_{0}\right), u_{3}\left(s_{0}\right), z\left(s_{0}\right)\right) \in \partial \Psi_{3}$, then $u_{2}^{\prime}\left(s_{0}\right)=g_{1}\left(u_{3}\left(s_{0}\right)\right) /(b c)>0$. Since $E_{1}$ is equilibrium, in summary, $\Psi$ is positive invariant.

Since $\lambda_{1}>\lambda_{2}>0$, stable manifold theorem implies that there exists a one-dimensional strong unstable manifold $\mathscr{W}_{1}$ tangent to $e_{1}$ at $E_{1}$ such that the point on $\mathscr{W}_{1}$ near $E_{1}$ can be expressed by

$$
G_{1}(\varepsilon)=\varepsilon e_{1}+o(\varepsilon) .
$$

Furthermore, there is a two-dimensional unstable manifold $\mathscr{W}_{2}$ tangent to span $\left\{e_{1}, e_{2}\right\}$ at $E_{1}$ such that $\mathscr{W}_{2}$ near $E_{1}$ can be expressed by

$$
G_{2}\left(\varepsilon_{1}, \varepsilon_{2}\right)=\varepsilon_{1} e_{1}+\varepsilon_{2} e_{2}+o\left(\sqrt{\varepsilon_{1}^{2}+\varepsilon_{2}^{2}}\right)
$$

Lemma 9. Suppose that $u(s) \triangleq\left(u_{2}(s), u_{3}(s), z(s)\right)$ is a solution of (17) such that $u(0) \in \mathscr{W}_{1}$ for small $\varepsilon>0$. Then, $u(s)$ will leave $W$ and enter $P$.

Proof. Obviously, $u(s)$ satisfies initial condition (39) by the structure of $e_{1}$, and Lemma 8 implies $u(s)>0(u(s)>0$ means that $u_{i}(s)>0$ and $\left.z(s)>0, i=2,3\right)$ for every $s>0$.

Furthermore, Lemma 8 shows that $u_{3}^{\prime}(s)=z(s)>k u_{3}(s)$, implying $\lim _{s \rightarrow+\infty} u_{3}(s)=+\infty$. Since $u_{2}(s)<1 / b$, it follows $\lim _{s \rightarrow+\infty} z(s)=+\infty$. Suppose that $u_{2}(s)<u^{*}$ for every $s>0$. Then

$$
\begin{aligned}
u_{2}^{\prime} & >\frac{\left[\left(\left(f_{1}\left(u^{*}\right) / u^{*}\right)+\left(g_{1}\left(u_{3}\right) / u^{*}\right)\right)\left((1 / b)-u^{*}\right)-b\right] u_{2}}{c} \\
& >\frac{\left[\left(\left(f_{1}\left(u^{*}\right) / u^{*}\right)+\left(g_{1}\left(2 u^{*}\right) / u^{*}\right)\right)\left((1 / b)-u^{*}\right)-b\right] u_{2}}{c} \\
& =\frac{M u_{2}}{c}>0
\end{aligned}
$$

for large $s$ since $u_{3}(s)$ and $g_{1}\left(u_{3}\right)$ are strictly monotonous increasing with respect to $s$ and $u_{3}$, respectively. Thus, we have that $\lim _{s \rightarrow+\infty} u_{2}(s)=+\infty$, contradicting $u_{2}(s)<1 / b$ for any $s \in R$. Therefore, there exists $s_{1}>0$ such that $u_{2}\left(s_{1}\right)=u^{*}$. Without losing generality, let $s_{1}=\inf \left\{s>0: u_{2}(s)=u^{*}\right\}$. Obviously, we have $u_{2}^{\prime}\left(s_{1}\right) \geq 0$. If $u_{3}\left(s_{1}\right)<u^{*}$, then

$$
\begin{aligned}
u_{2}^{\prime} & \left(s_{1}\right) \\
& =\frac{\left[\left(\left(f_{1}\left(u^{*}\right) / u^{*}\right)+\left(g_{1}\left(u_{3}\left(s_{1}\right)\right) / u^{*}\right)\right)\left((1 / b)-u^{*}\right)-b\right] u^{*}}{c} \\
& <0,
\end{aligned}
$$

which is a contradiction. Therefore, $u_{3}\left(s_{1}\right) \geq u^{*}$ and $u\left(s_{1}\right) \in$ $\partial P$. Then, the construction of $W^{-}$shows that $u(s)$ will leave $W$ and enter $P$.

Let $C$ be a small circle on $\mathscr{W}_{2}$ centered at $E_{1}$. Then, points on $C$ can be expressed in terms of local coordinate by

$$
F(\theta) \triangleq G_{2}(\varepsilon \cos \theta, \varepsilon \sin \theta)=\varepsilon\left[e_{1} \cos \theta+e_{2} \sin \theta+O(\varepsilon)\right],
$$

where $\theta \in\left[\theta_{1}, 2 \pi+\theta_{1}\right), \varepsilon>0$, and $\theta_{1}$ is chosen such that $F\left(\theta_{1}\right)$ lies on $\mathscr{W}_{1}$ with $z>0$. Then, stable manifold theorem shows that $\theta_{1} \rightarrow 0$ when $\varepsilon \rightarrow 0$. Denote $F(\theta) \triangleq$ $\left(\bar{u}_{2}(\theta), \bar{u}_{3}(\theta), \bar{z}(\theta)\right)$.

Lemma 10. There exists $a \theta_{2} \in(\pi / 2,3 \pi / 4)$ such that

$$
\bar{z}\left(\theta_{2}\right)=0, \quad 0<\bar{u}_{3}\left(\theta_{2}\right)<\bar{u}_{2}\left(\theta_{2}\right)<u^{*},
$$

and that

$$
\bar{z}(\theta)>0, \quad 0<\bar{u}_{2}\left(\theta_{2}\right)<u^{*}, \quad 0<\bar{u}_{3}(\theta)<u^{*}
$$

for $\theta \in\left[\theta_{1}, \theta_{2}\right)$.

Proof. From (51), we have

$$
\begin{aligned}
\bar{z}(\theta)= & \varepsilon\left[\lambda_{1} \cos \theta+\lambda_{2} \sin \theta+O(\varepsilon)\right] \\
= & \varepsilon \sqrt{\lambda_{1}^{2}+\lambda_{2}^{2}} \\
& \times\left[\frac{\lambda_{1}}{\sqrt{\lambda_{1}^{2}+\lambda_{2}^{2}}} \cos \theta+\frac{\lambda_{2}}{\sqrt{\lambda_{1}^{2}+\lambda_{2}^{2}}} \sin \theta+O(\varepsilon)\right] \\
= & \varepsilon \sqrt{\lambda_{1}^{2}+\lambda_{2}^{2}}\left[\sin \left(\varphi_{0}+\theta\right)+O(\varepsilon)\right],
\end{aligned}
$$


where $\sin \left(\varphi_{0}\right)=\lambda_{1} / \sqrt{\lambda_{1}^{2}+\lambda_{2}^{2}}, \cos \left(\varphi_{0}\right)=\lambda_{2} / \sqrt{\lambda_{1}^{2}+\lambda_{2}^{2}}$, and $\varphi_{0} \in(\pi / 4, \pi / 2)$ since $\lambda_{1}>\lambda_{2}$. Therefore, $\bar{z}\left(\theta_{2}\right)=0$ and $\theta_{2} \epsilon$ $[0, \pi]$ imply that $\theta_{2}=\pi-\varphi_{0}+O(\varepsilon) \in(\pi / 2,3 \pi / 4)$. Obviously, $\bar{z}(\theta)>0$ for any $\theta \in\left[\theta_{1}, \theta_{2}\right)$. However,

$$
\begin{gathered}
\bar{u}_{2}(\theta)=\frac{\varepsilon g_{1}^{\prime}(0)}{b c}\left[\frac{\cos \theta}{\lambda_{1}-\lambda^{*}}+\frac{\sin \theta}{\lambda_{2}-\lambda^{*}}+O(\varepsilon)\right] \\
=\frac{\varepsilon}{m}\left[\left(-\lambda_{1}^{2}+\lambda_{1} c+m\right) \cos \theta\right. \\
\left.+\left(-\lambda_{2}^{2}+\lambda_{2} c+m\right) \sin \theta+O(\varepsilon)\right], \\
\bar{u}_{3}(\theta)=\varepsilon[\cos \theta+\sin \theta+O(\varepsilon)],
\end{gathered}
$$

$\bar{u}_{2}(\theta)-\bar{u}_{3}(\theta)=\frac{\varepsilon}{m}\left[\lambda_{1}\left(c-\lambda_{1}\right) \cos \theta+\lambda_{2}\left(c-\lambda_{2}\right) \sin \theta+O(\varepsilon)\right]$.

Then, equality $\bar{z}\left(\theta_{2}\right)=\varepsilon\left[\lambda_{1} \cos \theta_{2}+\lambda_{2} \sin \theta_{2}+O(\varepsilon)\right]=0$, together with the last of (55), reveals $\bar{u}_{2}\left(\theta_{2}\right)-\bar{u}_{3}\left(\theta_{2}\right)=\varepsilon\left[\left(\lambda_{1}-\right.\right.$ $\left.\left.\lambda_{2}\right) \lambda_{2} \sin \theta_{2}+O(\varepsilon)\right] / m>0$; that is, $\bar{u}_{2}\left(\theta_{2}\right)>\bar{u}_{3}\left(\theta_{2}\right)$. For $\theta \in$ $\left[\theta_{1}, \theta_{2}\right]$, the first and second equalities of (55) imply that $0<$ $\bar{u}_{i}(\theta)<u^{*}$ where $i=2,3$ since $\lambda_{1}>\lambda_{2}$ and $0<\varepsilon \ll 1$.

\section{Let}

$$
\Sigma=\left\{F(\theta) \mid \theta \in\left[\theta_{1}, \theta_{2}\right], \varepsilon \text { is small enough }\right\} .
$$

By Lemma $10, \Sigma$ is an arc of circle, $\Sigma \subseteq W$, and the solution of (17) with initial value being the endpoint $F\left(\theta_{2}\right)$ will enter $Q$ since $F\left(\theta_{2}\right) \in W^{-} \cap \partial Q$. From Lemma 9, the solution of (17) with initial value being the endpoint $F\left(\theta_{1}\right)$ will enter $P$.

\subsection{Traveling Wave Solution for $c>c^{*}$}

Lemma 11. Let $u(s)=\left(u_{2}(s), u_{3}(s), z(s)\right)$ be a solution of (17) such that $u(0) \in \Lambda$. If $u(s) \in W$ for any $s \geq 0$, then $u(s) \in \Lambda$ for any $s>0$, where

$$
\Lambda=\left\{\left(u_{2}, u_{3}, z\right): 0<u_{2}<u^{*}, u_{3}>0,0<z<k u_{3}\right\}
$$

and $k=c+\sqrt{c^{2}+4 m}$.

Proof. Set $s_{0}=\inf \{s: u(s) \notin \Lambda, s \geq 0\}$. Suppose the conclusion is false; that is, $s_{0}<+\infty$. Obviously, $s_{0}>0$ and $u\left(s_{0}\right) \in$ $\partial \Lambda$ where

$$
\begin{aligned}
& \partial \Lambda=\left(\cup_{i=1}^{7} \partial \Lambda_{i}\right) \cup E_{2}, \\
& \partial \Lambda_{1}=\left\{\left(u_{2}, u_{3}, z\right): u_{2}=u^{*}, u_{3} \geq u^{*}, 0 \leq z \leq k u_{3}\right\} \backslash E_{2}, \\
& \partial \Lambda_{2}=\left\{\left(u_{2}, u_{3}, z\right): 0<u_{3} \leq u_{2} \leq u^{*}, z=0\right\} \backslash E_{2}, \\
& \partial \Lambda_{3}=\left\{\left(u_{2}, u_{3}, z\right): u_{2}=0, u_{3}>0,0 \leq z \leq k u_{3}\right\}, \\
& \partial \Lambda_{4}=\left\{\left(u_{2}, u_{3}, z\right): u_{2}=u^{*}, 0<u_{3}<u^{*}, 0<z \leq k u_{3}\right\}, \\
& \partial \Lambda_{5}=\left\{\left(u_{2}, u_{3}, z\right): 0 \leq u_{2} \leq u^{*}, u_{3}>0, z=k u_{3}\right\}, \\
& \partial \Lambda_{6}=\left\{\left(u_{2}, u_{3}, z\right): u_{3}>u_{2}, 0 \leq u_{2}<u^{*}, u_{3}>0, z=0\right\}, \\
& \partial \Lambda_{7}=\left\{\left(u_{2}, u_{3}, z\right): 0 \leq u_{2} \leq u^{*}, u_{3}=z=0\right\} .
\end{aligned}
$$

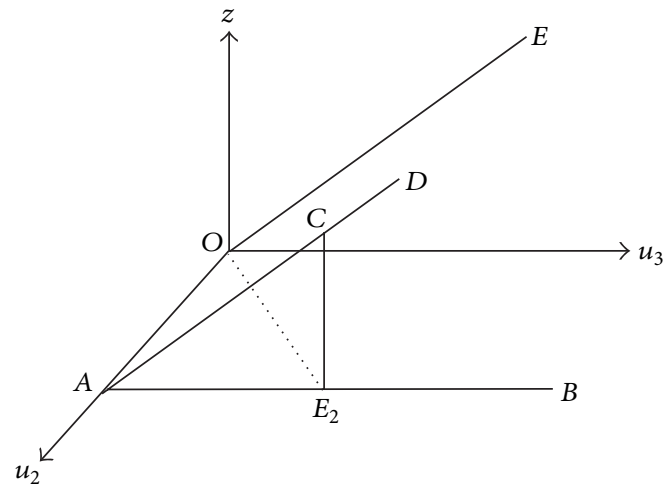

FIGURE 3: The construction of $\partial \Lambda$.

In Figure 3, we find $\partial \Lambda_{1}=\left\{\right.$ unbounded area $\left.B E_{2} C D\right\}$, $\partial \Lambda_{2}=$ triangle $\left.O A E_{2} O\right\}, \partial \Lambda_{3}=$ unbounded cone $\left.u_{3} O E\right\}, \partial \Lambda_{4}=$ triangle $\left.A E_{2} C A\right\}, \partial \Lambda_{5}=$ unbounded area $D C A O E\}, \partial \Lambda_{6}=$ unbounded area $\left.B E_{2} O u_{3}\right\}$, and $\partial \Lambda_{7}=$ $\{$ segment $O A\}$.

Since $\partial \Lambda_{1} \cup \partial \Lambda_{2} \subset W^{-}$, thus $u\left(s_{0}\right) \notin \partial \Lambda_{1} \cup \partial \Lambda_{2}$. If $u\left(s_{0}\right) \epsilon$ $\partial \Lambda_{3}$, we have $u_{2}^{\prime}\left(s_{0}\right) \leq 0$ because $u_{2}(s)>0$ for $0<s<s_{0}$ and $u_{2}\left(s_{0}\right)=0$. However, $u_{2}^{\prime}\left(s_{0}\right)=g_{1}\left(u_{3}\left(s_{0}\right)\right) /(b c)>0$ which is a contradiction. Therefore, $u\left(s_{0}\right) \notin \partial \Lambda_{3}$. If $u\left(s_{0}\right) \in \partial \Lambda_{4}$, then

$$
\begin{aligned}
& u_{2}^{\prime}\left(s_{0}\right) \\
& \quad=\frac{\left[\left(\left(f_{1}\left(u^{*}\right) / u^{*}\right)+\left(g_{1}\left(u_{3}\left(s_{0}\right)\right) / u^{*}\right)\right)\left((1 / b)-u^{*}\right)-b\right] u^{*}}{c} \\
& \quad<0,
\end{aligned}
$$

contradicting $u_{2}^{\prime}\left(s_{0}\right) \geq 0$. If $u\left(s_{0}\right) \in \partial \Lambda_{5}$, then

$$
\begin{aligned}
& {\left[z(s)-k u_{3}(s)\right]_{s=s_{0}}^{\prime}} \\
& \quad=(c-k) z\left(s_{0}\right)+m\left[u_{3}\left(s_{0}\right)-u_{2}\left(s_{0}\right)\right] \\
& \quad=[(c-k) k+m] u_{3}\left(s_{0}\right)-m u_{2}\left(s_{0}\right)<0
\end{aligned}
$$

since $(c-k) k+m<0$, contradicting $\left[z(s)-k u_{3}(s)\right]_{s=s_{0}}^{\prime} \geq 0$. If $u\left(s_{0}\right) \in \partial \Lambda_{6}$, then $z^{\prime}\left(s_{0}\right)=m\left[u_{3}\left(s_{0}\right)-u_{2}\left(s_{0}\right)\right]>0$ which is a contradiction. In conclusion, $u\left(s_{0}\right) \notin \partial \Lambda_{4} \cup \partial \Lambda_{5} \cup \partial \Lambda_{6}$. If $u\left(s_{0}\right) \in \partial \Lambda_{7}$, then $u_{3}(s)>0$ and $z(s)>0$ for any $0<$ $s<s_{0}$. Hence, $u_{3}^{\prime}(s)=z(s)>0$ for any $0<s<s_{0}$, which implies that $u_{3}\left(s_{0}\right)>u_{3}(0)>0$. From this contradiction we find $u\left(s_{0}\right) \notin \partial \Lambda_{7}$. Because $E_{2}$ is a constant solution, we get $u\left(s_{0}\right) \neq E_{2}$. In summary, $u\left(s_{0}\right) \notin \partial \Lambda$ and $s_{0}=+\infty$. The proof is completed.

Lemma 12. There exists a point $u_{0}=\left(u_{20}, u_{30}, z_{0}\right) \in \sum$ such that the solution $u\left(s ; u_{0}\right)=\left(u_{2}(s), u_{3}(s), z(s)\right)$ of (17) with initial value being $u_{0}$ will stay in $W$ for any $s>0$.

Proof. It is sufficient to prove $\Sigma \neq \Sigma_{0}$. Suppose that $\Sigma=$ $\Sigma_{0}$. Firstly, we verify Conditions (1) and (2) of Lemma 6. Condition (1) of Lemma 6 is valid since $W$ is closed. 
Suppose $u_{0}=\left(u_{20}, u_{30}, z_{0}\right) \in \Sigma, s<T\left(u_{0}\right)$ and $u\left(s ; u_{0}\right) \epsilon$ $W \backslash W^{-}$. Then, $u\left(s ; u_{0}\right) \in$ int $W \cup J$ and $u_{0} \neq F\left(\theta_{2}\right)$ since $F\left(\theta_{2}\right) \in W^{-}$. The structure of $\sum$ implies that $u_{20}>0, u_{30}>0$, and $z_{0}>0$. By the proof of Lemma 11, we have that $u\left(s ; u_{0}\right)>$ 0 for $s<T\left(u_{0}\right)$. Therefore, $u\left(s ; u_{0}\right) \notin J$ and $u\left(s ; u_{0}\right) \in \operatorname{int} W$. Condition (2) of Lemma 6 holds.

Lemma 6 shows that $\Sigma$ is homeomorphic to $H(\Sigma)$. Since

$$
H\left(F\left(\theta_{1}\right)\right) \in \partial P \cap W^{-}, \quad H\left(F\left(\theta_{2}\right)\right) \in \partial Q \cap W^{-},
$$

and $W^{-}$is disconnected, we have that $H(\Sigma)$ is disconnected, contradicting the connection of $\Sigma$. Thus, $\Sigma \neq \Sigma_{0}$ and the proof is completed.

Lemma 13. Let $c>c^{*}$. Then, there exists a positive solution $u(s)=\left(u_{2}(s), u_{3}(s), z(s)\right)$ of (17) such that

$$
u(+\infty)=E_{2}, \quad u(-\infty)=E_{1} .
$$

Proof. By Lemma 12 there exists a point $u_{0}=\left(u_{20}, u_{30}, z_{0}\right) \in$ $\Sigma$ such that the solution $u\left(s ; u_{0}\right)=\left(u_{2}(s), u_{3}(s), z(s)\right)$ of (17) with initial value being $u_{0}$ will stay in $W$ for any $s>0$. Furthermore, Lemma 11 shows $u\left(s ; u_{0}\right)>0$ for any $s \geq 0$. Stable manifold theorem implies that $u\left(s ; u_{0}\right)>0$ for any $s \leq 0$ and $\lim _{s \rightarrow-\infty} u\left(s ; u_{0}\right)=E_{1}$. Therefore, $u\left(s ; u_{0}\right)$ is a positive solution.

To complete the proof, it is sufficient to show that $\lim _{s \rightarrow+\infty} u\left(s ; u_{0}\right)=E_{2}$. By Lemma 11, we know that $u_{2}(s)<$ $u^{*}$ for any $s>0$ since $u\left(s ; u_{0}\right)$ remains in $W$ for all $s$. Because $u_{3}^{\prime}(s)=z(s)>0$, then the limit of $u_{3}(s)$ exists; that is, $\lim _{s \rightarrow+\infty} u_{3}(s)=u_{3}^{*}$ and $0<u_{3}^{*} \leq+\infty$. Suppose that $u^{*}<u_{3}^{*} \leq+\infty$. The first equation of (17) shows that

$$
\begin{aligned}
u_{2}^{\prime} & >\frac{\left[\left(\left(f_{1}\left(u^{*}\right) / u^{*}\right)+\left(g_{1}\left(u_{3}\right) / u^{*}\right)\right)\left((1 / b)-u^{*}\right)-b\right] u_{2}}{c} \\
& >\left(\left[\left(\frac{f_{1}\left(u^{*}\right)}{u^{*}}+\frac{g_{1}\left(\left(u_{3}^{*}+u^{*}\right) / 2\right)}{u^{*}}\right)\left(\frac{1}{b}-u^{*}\right)-b\right] u_{2}\right) \times(c)^{-1} \\
& =\frac{M u_{2}}{c}>0
\end{aligned}
$$

for large $s$, which implies that there is an $s^{*}>0$ such that $u_{2}\left(s^{*}\right)>u^{*}$. This is a contradiction, and thus $0<u_{3}^{*} \leq u^{*}$. From the first equation of (17), we have $\lim _{s \rightarrow+\infty} u_{2}(s)=u_{2}^{*}$ where $u_{2}^{*}$ is the only positive root of algebra equation

$$
\left[f_{1}\left(u_{2}\right)+g_{1}\left(u_{3}^{*}\right)\right]\left(\frac{1}{b}-u_{2}\right)-b u_{2}=0 .
$$

At the same time, the third equation of (17) implies $\lim _{s \rightarrow+\infty} z(s)=z^{*}$ and $z^{*}=m\left(u_{2}^{*}-u_{3}^{*}\right) / c$ or $\pm \infty$. It is impossible that $z^{*}= \pm \infty$ due to the boundedness of $u_{3}(s)$. In conclusion, the $\operatorname{limit}_{\lim _{s \rightarrow+\infty}} u(s)=\left(u_{2}^{*}, u_{3}^{*}, z^{*}\right)$ exists and is finite. By [42], $\left(u_{2}^{*}, u_{3}^{*}, z^{*}\right)$ must be equilibrium. Since $u_{3}^{*}>0$, then $\left(u_{2}^{*}, u_{3}^{*}, z^{*}\right)=E_{2}$.

Noticing the relation of systems (17) and (10) completes the proof of Theorem 1 for case $c>c^{*}$.
5.3. Traveling Wave Solution for $c=c^{*}$. Firstly, suppose $c>$ $c^{*}$ and let $u\left(s ; u_{0}\right)=\left(u_{2}(s), u_{3}(s), z(s)\right)$ be the traveling wave solution of (17). Then, Lemma 11 implies that $u\left(s ; u_{0}\right) \in \Lambda$ for all $s$. From the proof of Lemma 13, we find $u_{3}(s) \leq u^{*}$. Therefore, for all $s$, we have $u\left(s ; u_{0}\right) \in \Pi$ where

$$
\Pi=\left\{\left(u_{2}, u_{3}, z\right): 0<u_{2}<u^{*}, 0<u_{3} \leq u^{*}, 0<z<k u_{3}\right\} .
$$

Let $\left\{c_{n}\right\}$ be a sequence such that $c^{*}<c_{n}<c_{n+1}$ for any $n$ and $\lim _{n \rightarrow \infty} c_{n}=c^{*}$. Set $k_{n}=c_{n}+\sqrt{c_{n}^{2}+4 m}$ and

$\Pi_{n}=\left\{\left(u_{2}, u_{3}, z\right): 0<u_{2} \leq u^{*}, 0<u_{3} \leq u^{*}, 0<z \leq k_{n} u_{3}\right\}$.

Then, $\Pi_{n} \subseteq \Pi_{1}$ for any $n$.

Lemma 13 shows that there is a positive solution $w_{n}(s)=$ $\left(u_{2, n}(s), u_{3, n}(s), z_{n}(s)\right)$ for system

$$
\begin{gathered}
u_{2}^{\prime}=\frac{\left[\left(f_{1}\left(u_{2}\right)+g_{1}\left(u_{3}\right)\right)\left((1 / b)-u_{2}\right)-b u_{2}\right]}{c_{n}}, \\
u_{3}^{\prime}=z, \\
z^{\prime}=c_{n} z+m\left(u_{3}-u_{2}\right),
\end{gathered}
$$

satisfying boundary condition (62) such that $w_{n}(s) \in \Pi_{n} \subseteq$ $\Pi_{1}$ for any $s$.

Lemma 14. Let $c=c^{*}$. Then, there exists a traveling wave solution for system (6) satisfying boundary condition (11).

Proof. It is sufficient to prove that there exists a positive solution $u(s)=\left(u_{2}(s), u_{3}(s), z(s)\right)$ of (17) satisfying boundary condition (62).

Firstly, we show that sequences $\left\{u_{2, n}\right\},\left\{u_{3, n}\right\},\left\{z_{n}\right\},\left\{u_{2, n}^{\prime}\right\}$, $\left\{u_{3, n}^{\prime}\right\}$, and $\left\{z_{n}^{\prime}\right\}$ are uniformly bounded and equicontinuous. The idea of Lemma 11 in [34] is used. Obviously, $\left\{u_{2, n}\right\},\left\{u_{3, n}\right\}$, and $\left\{z_{n}\right\}$ are uniformly bounded since $w_{n}(s) \subseteq \Pi_{1}$ for any $s$. Because $w_{n}(s)=\left(u_{2, n}(s), u_{3, n}(s), z_{n}(s)\right)$ is the solution of (67), $\left\{u_{2, n}^{\prime}\right\},\left\{u_{3, n}^{\prime}\right\}$, and $\left\{z_{n}^{\prime}\right\}$ are also uniformly bounded. Since $\left|z_{n}\left(s_{1}\right)-z_{n}\left(s_{2}\right)\right|=z_{n}^{\prime}\left(s_{3}\right)\left|s_{1}-s_{2}\right|$ where $s_{1}<s_{3}<s_{2}$, then $\left\{z_{n}\right\}$ is equicontinuous. Similarly, $\left\{u_{2, n}\right\}$ and $\left\{u_{3, n}\right\}$ are also equicontinuous. By differentiating the equations of (67) and using the previous bounds, we can get that $\left\{u_{2, n}^{\prime \prime}\right\},\left\{u_{3, n}^{\prime \prime}\right\}$, and $\left\{z_{n}^{\prime \prime}\right\}$ are uniformly bounded, and hence $\left\{u_{2, n}^{\prime}\right\},\left\{u_{3, n}^{\prime}\right\}$, and $\left\{z_{n}^{\prime}\right\}$ are equicontinuous.

The previous paragraph and Arzelà-Ascoli theorem imply that there exist subsequences, again denoted by $\left\{u_{2, n}\right\},\left\{u_{3, n}\right\}$, and $\left\{z_{n}\right\}$ and functions $u_{2}, u_{3}$, and $z$ such that

$$
u_{2, n} \longrightarrow u_{2}, \quad u_{3, n} \longrightarrow u_{3}, \quad z_{n} \longrightarrow z
$$

uniformly on compact subsets of $\mathbb{R}$, thus pointwise on $\mathbb{R}$. Same arguments imply that $\left\{u_{2, n}^{\prime}\right\},\left\{u_{3, n}^{\prime}\right\}$, and $\left\{z_{n}^{\prime}\right\}$ are also uniformly convergent on compact subsets of $\mathbb{R}$ and pointwise convergent on $\mathbb{R}$. Consequently, we get

$$
u_{2, n}^{\prime} \rightarrow u_{2}^{\prime}, \quad u_{3, n}^{\prime} \longrightarrow u_{3}^{\prime}, \quad z_{n}^{\prime} \longrightarrow z^{\prime}
$$



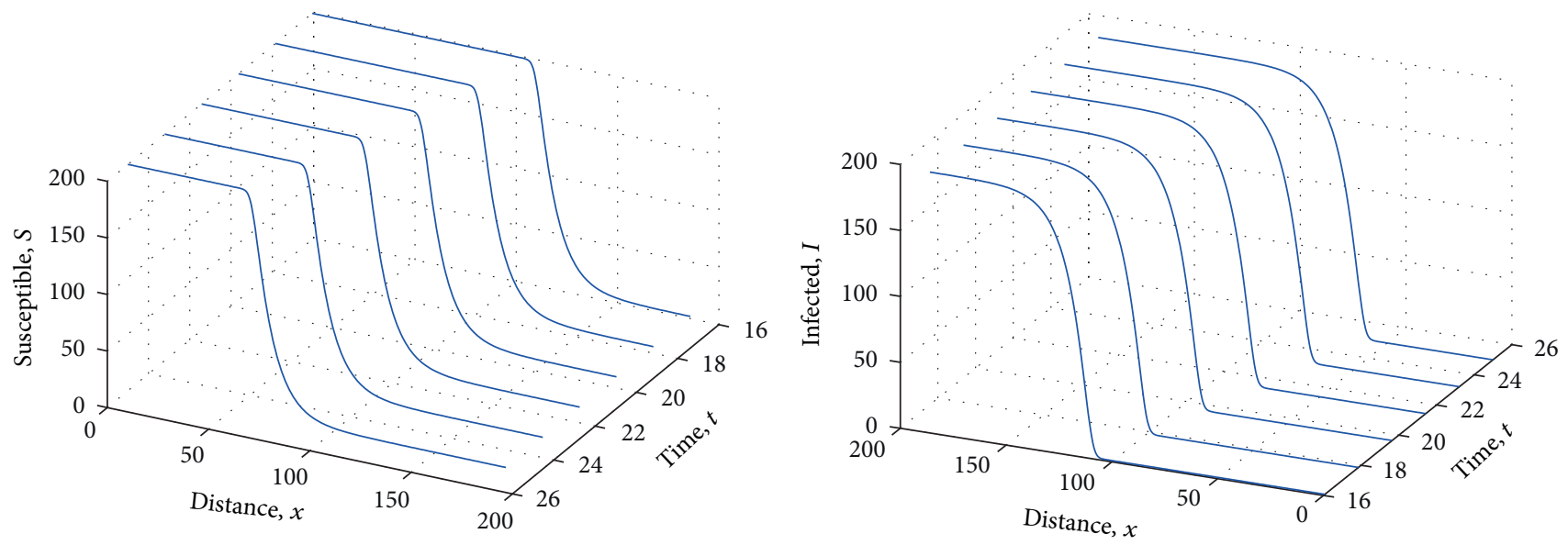

FIgURE 4: The wave profiles for $S$ and $I$ and their movements.

Since $\left(u_{2, n}, u_{3, n}, z_{n}\right)$ is the solution of (67), then $u(s)=$ $\left(u_{2}(s), u_{3}(s), z(s)\right)$ is the solution of (17) for $c=c^{*}$ and $u(s) \in$ $\operatorname{cl}\left(\Pi_{1}\right)$, where $\operatorname{cl}\left(\Pi_{1}\right)$ is the closer of $\Pi_{1}$. Because system $(67)$ is autonomous and $\left(u_{2, n}, u_{3, n}, z_{n}\right)$ satisfies boundary condition (62), we can assume that $u_{3, n}(0)=u^{*} / 2$ for all $n$; thus, $u_{3}(0)>$ 0 . Then, similar to the proof of Lemma 13, we have that the solution $u(s)$ satisfies boundary condition (62).

\section{Simulations}

In this section, we present some simulations to confirm the theoretical results. Set

$$
f(I)=\frac{\beta_{1} I}{K_{h}+I}, \quad g(B)=\frac{\beta_{2} B}{K_{e}+B}
$$

and assign numerical values to parameters as follows:

$$
\begin{gathered}
b=0.01, \quad e=1, \quad m=0.5, \quad K_{e}=6, \quad K_{h}=2, \\
\beta_{1}=0.62, \quad \beta_{2}=0.001, \quad N=200, \quad d=2 .
\end{gathered}
$$

Obviously, such selection for $f(I)$ and $g(B)$ satisfies (A1) and (A2). Then, the traveling wave solution is described in Figure 4.

\section{Conflict of Interests}

The authors declare that there is no conflict of interests regarding the publication of this paper.

\section{Acknowledgments}

The authors are supported by the Fundamental Research Funds for the Central Universities (Grants nos. XDJK2012C042 and SWU113048) and NSFC (Grant no. $11201380)$.

\section{References}

[1] Centers for Disease Control and Prevention, USA, http://www .cdc.gov/cholera/general/.

[2] World Health Organization, http://www.who.int/csr/don/ archive/disease/cholera/en/.

[3] V. Capasso and S. L. Paveri-Fontana, "Mathematical model for the 1973 cholera epidemic in the European Mediterranean region," Revue d'Epidemiologie et de Sante Publique, vol. 27, no. 2, pp. 121-132, 1979.

[4] C. T. Codeço, "Endemic and epidemic dynamics of cholera: the role of the aquatic reservoir," BMC Infectious Diseases, vol. 1, no. 1, article 1, 2001.

[5] J. R. Andrews and S. Basu, "Transmission dynamics and control of cholera in Haiti: an epidemic model," The Lancet, vol. 377, no. 9773, pp. 1248-1255, 2011.

[6] K. T. Goh, S. H. Teo, S. Lam, and M. K. Ling, "Person-to-person transmission of cholera in a psychiatric hospital," Journal of Infection, vol. 20, no. 3, pp. 193-200, 1990.

[7] A. A. Weil, A. I. Khan, F. Chowdhury et al., "Clinical outcomes in household contacts of patients with cholera in Bangladesh," Clinical Infectious Diseases, vol. 49, no. 10, pp. 1473-1479, 2009.

[8] J. H. Tien and D. J. D. Earn, "Multiple transmission pathways and disease dynamics in a waterborne pathogen model," Bulletin of Mathematical Biology, vol. 72, no. 6, pp. 1506-1533, 2010.

[9] Z. Mukandavire, S. Liao, J. Wang, H. Gaff, D. L. Smith, and J. G. Morris Jr., "Estimating the reproductive numbers for the 2008-2009 cholera outbreaks in Zimbabwe," Proceedings of the National Academy of Sciences of the United States of America, vol. 108, no. 21, pp. 8767-8772, 2011.

[10] J. P. Tian and J. Wang, "Global stability for cholera epidemic models," Mathematical Biosciences, vol. 232, no. 1, pp. 31-41, 2011.

[11] D. M. Hartley, J. G. Morris Jr., and D. L. Smith, "Hyperinfectivity: a critical element in the ability of V. cholerae to cause epidemics?" PLoS Medicine, vol. 3, no. 1, pp. 63-69, 2006.

[12] Z. Shuai and P. van den Driessche, "Global dynamics of cholera models with differential infectivity," Mathematical Biosciences, vol. 234, no. 2, pp. 118-126, 2011.

[13] Z. Shuai, J. H. Tien, and P. van den Driessche, "Cholera models with hyperinfectivity and temporary immunity," Bulletin of Mathematical Biology, vol. 74, no. 10, pp. 2423-2445, 2012. 
[14] M. A. Jensen, S. M. Faruque, J. J. Mekalanos, and B. R. Levin, "Modeling the role of bacteriophage in the control of cholera outbreaks," Proceedings of the National Academy of Sciences of the United States of America, vol. 103, no. 12, pp. 4652-4657, 2006.

[15] R. P. Sanches, C. P. Ferreira, and R. A. Kraenkel, "The role of immunity and seasonality in cholera epidemics," Bulletin of Mathematical Biology, vol. 73, no. 12, pp. 2916-2931, 2011.

[16] A. Mutreja, D. W. Kim, N. R. Thomson et al., "Evidence for several waves of global transmission in the seventh cholera pandemic," Nature, vol. 477, no. 7365, pp. 462-465, 2011.

[17] R. R. Colwell, "Global climate and infectious disease: the cholera paradigm,” Science, vol. 274, no. 5295, pp. 2025-2031, 1996.

[18] S. M. Faruque, M. J. Islam, Q. S. Ahmad et al., "Self-limiting nature of seasonal cholera epidemics: role of host-mediated amplification of phage," Proceedings of the National Academy of Sciences of the United States of America, vol. 102, no. 17, pp. 61196124, 2005.

[19] S. Aniţa and V. Capasso, "Stabilization of a reaction-diffusion system modelling a class of spatially structured epidemic systems via feedback control," Nonlinear Analysis. Real World Applications, vol. 13, no. 2, pp. 725-735, 2012.

[20] V. Capasso, "Asymptotic stability for an integro-differential reaction-diffusion system," Journal of Mathematical Analysis and Applications, vol. 103, no. 2, pp. 575-588, 1984.

[21] V. Capasso and K. Kunisch, "A reaction-diffusion system arising in modelling man-environment diseases," Quarterly of Applied Mathematics, vol. 46, no. 3, pp. 431-450, 1988.

[22] V. Capasso and L. Maddalena, "Convergence to equilibrium states for a reaction-diffusion system modelling the spatial spread of a class of bacterial and viral diseases," Journal of Mathematical Biology, vol. 13, no. 2, pp. 173-184, 1981-1982.

[23] V. Capasso and R. E. Wilson, "Analysis of a reaction-diffusion system modeling man-environment-man epidemics," SIAM Journal on Applied Mathematics, vol. 57, no. 2, pp. 327-346, 1997.

[24] E. Bertuzzo, S. Azaele, A. Maritan, M. Gatto, I. RodriguezIturbe, and A. Rinaldo, "On the space-time evolution of a cholera epidemic," Water Resources Research, vol. 44, no. 1, Article ID W01424, 2008.

[25] E. Bertuzzo, R. Casagrandi, M. Gatto, I. Rodriguez-Iturbe, and A. Rinaldo, "On spatially explicit models of cholera epidemics," Journal of the Royal Society Interface, vol. 7, no. 43, pp. 321-333, 2010.

[26] L. Mari, E. Bertuzzo, L. Righetto et al., "Modelling cholera epidemics: the role of waterways, human mobility and sanitation," Journal of the Royal Society Interface, vol. 9, no. 67, pp. 376-388, 2012.

[27] O. Diekmann, "Thresholds and travelling waves for the geographical spread of infection," Journal of Mathematical Biology, vol. 6, no. 2, pp. 109-130, 1978.

[28] M. Lewis, J. Rencławowicz, and P. van den Driessche, "Traveling waves and spread rates for a West Nile virus model," Bulletin of Mathematical Biology, vol. 68, no. 1, pp. 3-23, 2006.

[29] J. Radcliffe and L. Rass, "The asymptotic speed of propagation of the deterministic nonreducible $n$-type epidemic," Journal of Mathematical Biology, vol. 23, no. 3, pp. 341-359, 1986.

[30] X.-Q. Zhao and W. Wang, "Fisher waves in an epidemic model," Discrete and Continuous Dynamical Systems B, vol. 4, no. 4, pp. 1117-1128, 2004.

[31] D. Xu and X.-Q. Zhao, "Bistable waves in an epidemic model," Journal of Dynamics and Differential Equations, vol. 16, no. 3, pp. 679-707, 2004.
[32] Y. Jin and X.-Q. Zhao, "Bistable waves for a class of cooperative reaction-diffusion systems," Journal of Biological Dynamics, vol. 2, no. 2, pp. 196-207, 2008.

[33] C.-H. Hsu and T.-S. Yang, "Existence, uniqueness, monotonicity and asymptotic behaviour of travelling waves for epidemic models," Nonlinearity, vol. 26, no. 1, pp. 121-139, 2013.

[34] S. R. Dunbar, "Travelling wave solutions of diffusive LotkaVolterra equations," Journal of Mathematical Biology, vol. 17, no. 1, pp. 11-32, 1983.

[35] S. R. Dunbar, "Traveling wave solutions of diffusive LotkaVolterra equations: a heteroclinic connection in $R^{4}$," Transactions of the American Mathematical Society, vol. 286, no. 2, pp. 557-594, 1984.

[36] W.-T. Li and S.-L. Wu, "Traveling waves in a diffusive predatorprey model with Holling type-III functional response," Chaos, Solitons \& Fractals, vol. 37, no. 2, pp. 476-486, 2008.

[37] C.-H. Hsu, C.-R. Yang, T.-H. Yang, and T.-S. Yang, "Existence of traveling wave solutions for diffusive predator-prey type systems," Journal of Differential Equations, vol. 252, no. 4, pp. 3040-3075, 2012.

[38] W. Huang, "Traveling wave solutions for a class of predator-prey systems," Journal of Dynamics and Differential Equations, vol. 24, no. 3, pp. 633-644, 2012.

[39] J. Huang, G. Lu, and S. Ruan, "Existence of traveling wave solutions in a diffusive predator-prey model," Journal of Mathematical Biology, vol. 46, no. 2, pp. 132-152, 2003.

[40] X. Lin, P. Weng, and C. Wu, "Traveling wave solutions for a predator-prey system with sigmoidal response function," Journal of Dynamics and Differential Equations, vol. 23, no. 4, pp. 903-921, 2011.

[41] R. S. Irving, Integers, Polynomials, and Rings, Springer, New York, NY, USA, 2004.

[42] Z. Ma and Y. Zhou, Qualitative and Stable Method of Ordinary Differential Equations, Science Press, Beijing, China, 2005. 


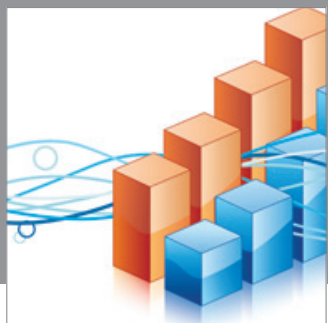

Advances in

Operations Research

mansans

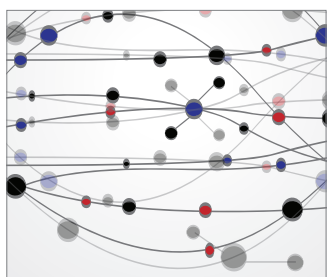

The Scientific World Journal
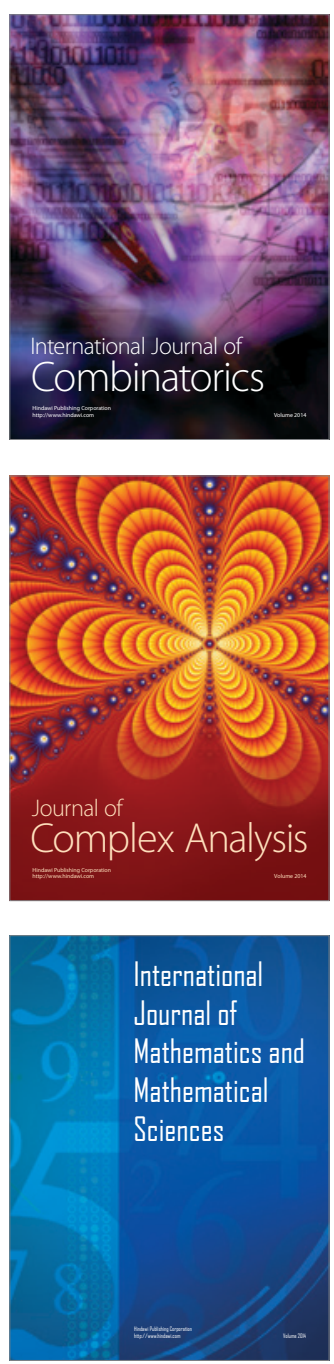
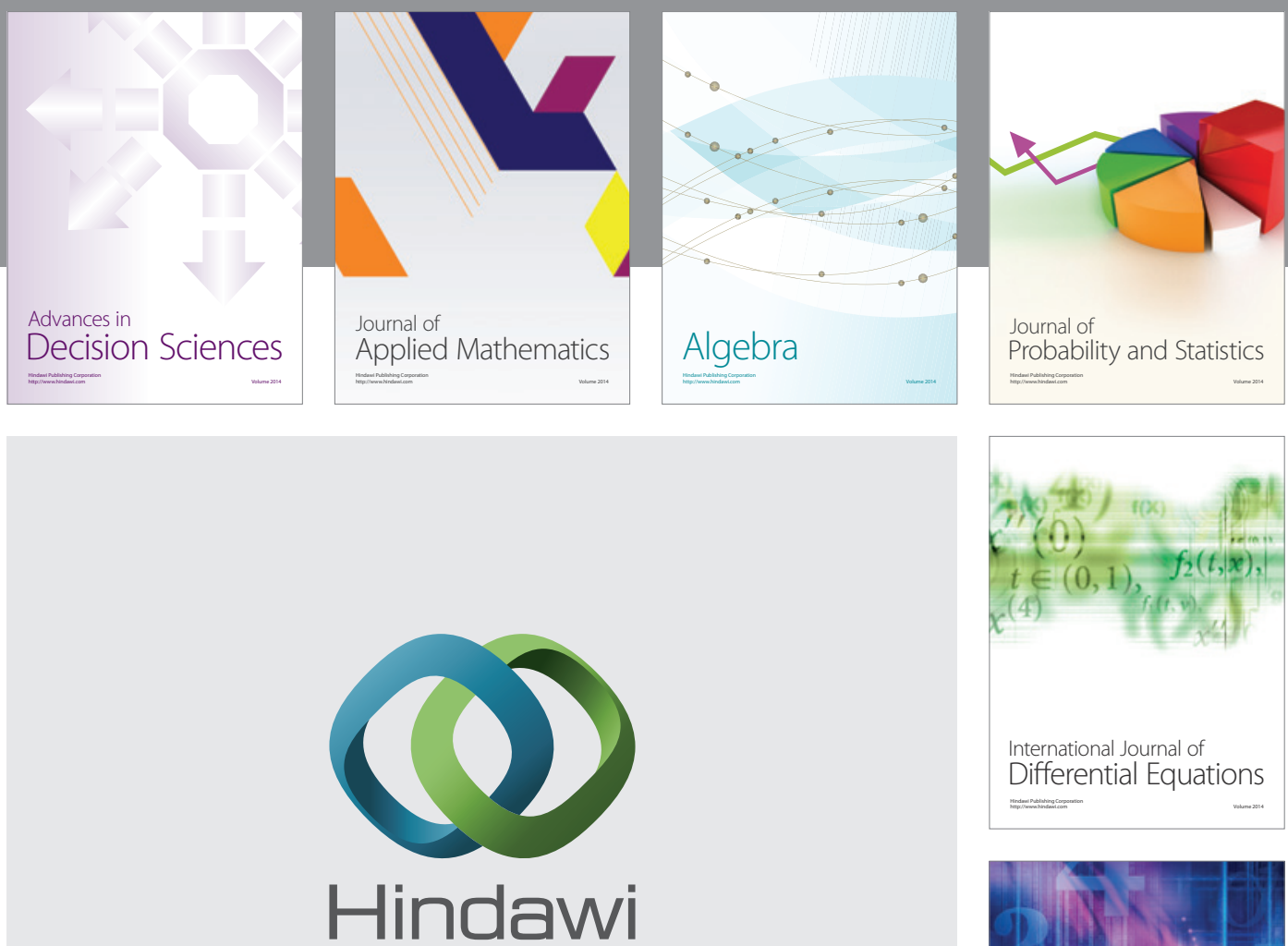

Submit your manuscripts at http://www.hindawi.com
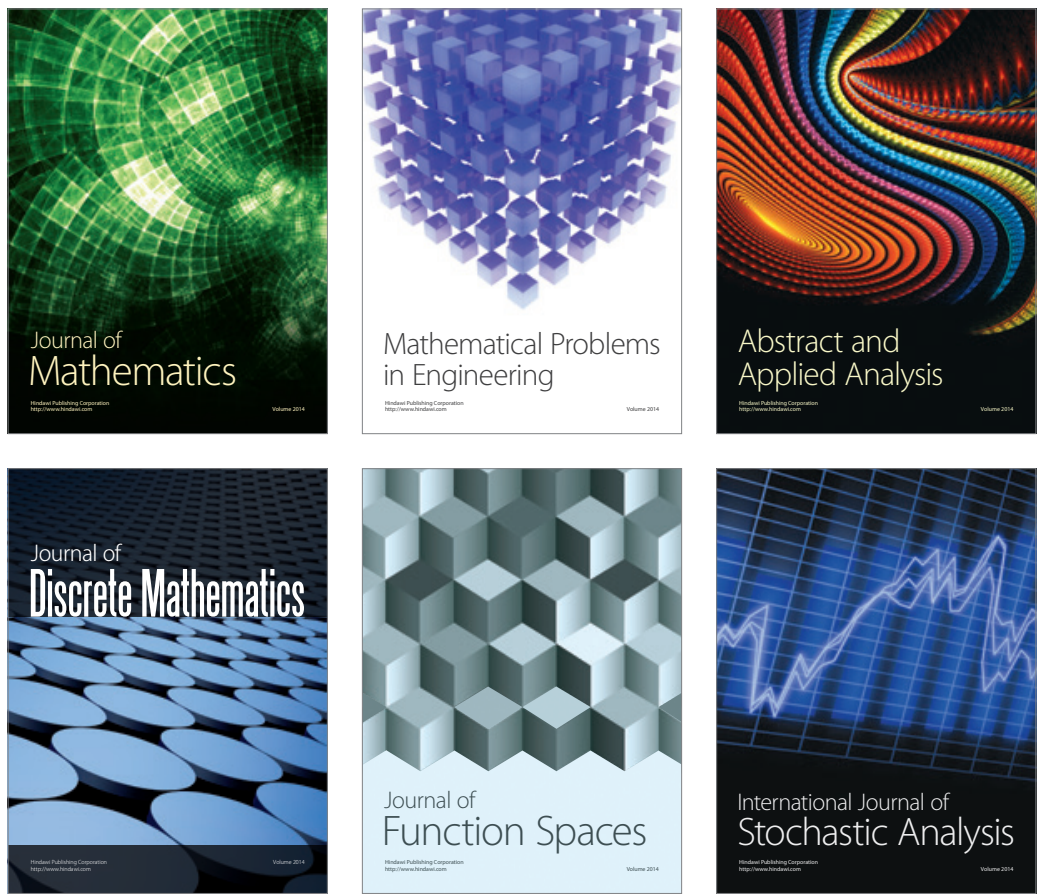

Journal of

Function Spaces

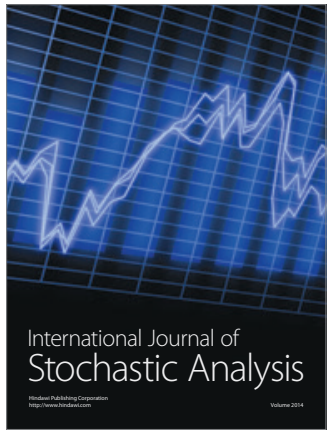

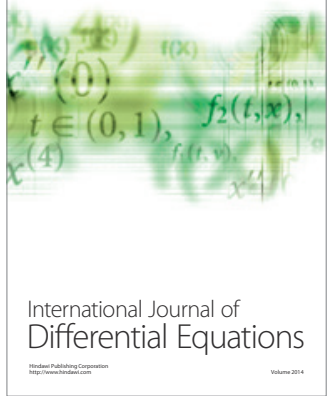
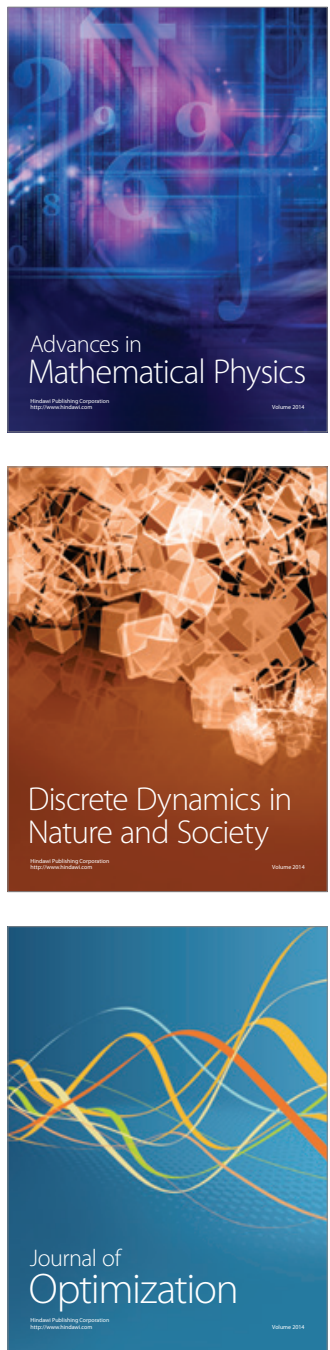